\title{
Optically Controlled Oscillators in an Engineered Bioelectric Tissue
}

\author{
Harold M. McNamara, ${ }^{1,2}$ Hongkang Zhang, ${ }^{3}$ Christopher A. Werley, ${ }^{3}$ and Adam E. Cohen ${ }^{1,3,4, *}$ \\ ${ }^{1}$ Department of Physics, Harvard University, 12 Oxford Street, Cambridge, Massachusetts 02138, USA \\ ${ }^{2}$ Harvard-MIT Division of Health Sciences and Technology, 12 Oxford Street, \\ Cambridge, Massachusetts 02138, USA \\ ${ }^{3}$ Department of Chemistry and Chemical Biology, Harvard University, \\ 12 Oxford Street, Cambridge, Massachusetts 02138, USA \\ ${ }^{4}$ Howard Hughes Medical Institute, 12 Oxford Street, Cambridge, Massachusetts 02138, USA \\ (Received 12 April 2016; revised manuscript received 3 June 2016; published 1 July 2016)
}

\begin{abstract}
Complex electrical dynamics in excitable tissues occur throughout biology, but the roles of individual ion channels can be difficult to determine due to the complex nonlinear interactions in native tissue. Here, we ask whether we can engineer a tissue capable of basic information storage and processing, where all functional components are known and well understood. We develop a cell line with four transgenic components: two to enable collective propagation of electrical waves and two to enable optical perturbation and optical readout of membrane potential. We pattern the cell growth to define simple cellular ring oscillators that run stably for $>2 \mathrm{~h}\left(\sim 10^{4}\right.$ cycles $)$ and that can store data encoded in the direction of electrical circulation. Using patterned optogenetic stimulation, we probe the biophysical attributes of this synthetic excitable tissue in detail, including dispersion relations, curvature-dependent wave front propagation, electrotonic coupling, and boundary effects. We then apply the biophysical characterization to develop an optically reconfigurable bioelectric oscillator. These results demonstrate the feasibility of engineering bioelectric tissues capable of complex information processing with optical input and output.
\end{abstract}

DOI: 10.1103/PhysRevX.6.031001

\section{INTRODUCTION}

Electrical dynamics in the brain, heart, endometrium, and pancreas arise from complex interactions among a large number of electrically excitable cells. The task of deciphering the relations between ion channel dynamics, single-cell properties, and tissue function is often daunting. A long-standing goal in synthetic biology is to design minimal systems that recreate aspects of naturally evolved variants [1]. By probing the necessity and sufficiency of individual components, this strategy can augment our understanding of more complex natural systems, and it can also point toward design principles for synthetic systems with novel behaviors and properties.

Most efforts within synthetic biology have focused on engineering intracellular networks to create basic functional elements such as oscillators [2], registers [3], and counters [4]. Multicellular interactions have the possibility to evince emergent dynamics not seen in individual cells [5]. Indeed, models of cellular automata have been a standard tool in theoretical explorations of complex networks. Excitable

*cohen@ chemistry.harvard.edu

Published by the American Physical Society under the terms of the Creative Commons Attribution 3.0 License. Further distribution of this work must maintain attribution to the author(s) and the published article's title, journal citation, and DOI.
Subject Areas: Biological Physics, Nonlinear Dynamics, Optics media have been shown theoretically capable of implementing complex computations [6,7], and light-sensitive Belousov-Zhabotinsky reactions have been experimentally used to implement rudimentary image transformations [8] and logical operations [9]. In the context of cardiac dynamics, simple models of coupled excitable cells show rich dynamics under periodic forcing, including period doubling and transitions to chaos in OD [10] and 1D [11], and spiral waves and wave breakup in 2D and 3D [12-14]. Experimental implementations of multicellular synthetic biological systems have remained challenging [5].

Hsu and co-workers showed that two voltage-gated ion channels were sufficient to induce electrical spikes in otherwise electrically inexcitable tumor cells [15]. Kirkton and Bursac used a similar strategy to engineer an electrically spiking cell line which additionally supported nearest-neighbor coupling via a gap junction protein [16]. Our lab then engineered a spiking cell line in which a genetically encoded fluorescent voltage indicator enabled direct, dye-free visualization of electrical waves [17].

While these systems constituted synthetic excitable tissues, their amenability to rigorous biophysical characterization and dynamical control was limited by a lack of spatiotemporal control over wave initiation. Spatially patterned optogenetic stimulation has been applied to study a variety of cardiac [18] and neuronal [19] preparations, but spectral cross talk of most optogenetic actuators and 
voltage reporters prevented simultaneous perturbation and measurement of membrane potential. We recently developed a genetic construct, called "Optopatch," which comprises a bicistronic vector for coexpression of a blue light-gated ion channel, CheRiff, and a red light-excited voltage indicator, QuasAr2. Together, these two proteins enabled cross-talk-free optical stimulation and measurement of membrane voltage in cultured neurons. However, the low brightness of QuasAr2 posed an obstacle to imaging with field of view sufficiently wide to study collective multicellular dynamics.

Here, we report a synthetic bioelectric tissue that supports simultaneous optical stimulation and readout of membrane voltage, and whose complex nonlinear dynamics enable basic information storage and processing. The tissue comprises human embryonic kidney (HEK-293) cells expressing an inward rectifier potassium channel $\left(\mathrm{K}_{\mathrm{ir}} 2.1\right)$, a voltagegated sodium channel $\left(\mathrm{Na}_{V} 1.5\right)$, and the Optopatch genes. To interact with this tissue, we developed an optical system engineered to combine high-precision patterned optogenetic stimulation with high-sensitivity voltage imaging over a large field of view $\left(5 \times 1 \mathrm{~mm}^{2}\right)$.

This high level of control enables biophysical characterization of the tissue at a previously unattainable level of detail and the creation of simple bioelectric circuits that could store and process information. Information storage is performed in bioelectric ring oscillators whose direction of circulation constitutes a topologically stable binary bit. Information processing is achieved via optical steering, gating, and modulation of electrical wave propagation. The combination of rationally designed excitable cells with high-resolution optogenetic control opens the door to creation of increasingly complex bioelectrical systems. While the biophysics of ion channels limits the speeds of these systems to $<100 \mathrm{~Hz}$, the living substrate opens the possibility for novel sensing [20] and tissue engineering applications [21], as well as fundamental studies of the biophysics of excitable tissues.

\section{EXCITABLE CELLS WITH A BIDIRECTIONAL OPTICAL INTERFACE}

HEK cells have a resting potential around $-20 \mathrm{mV}$, a membrane resistance $>500 \mathrm{M} \Omega$ [22], and on their own are electrically inexcitable. We engineer a HEK cell line that stably expresses an inward rectifier potassium channel $\mathrm{K}_{\mathrm{ir}} 2.1$ and a voltage-gated sodium channel $\mathrm{Na}_{V} 1.5$ [Fig. 1(a)]. The $\mathrm{K}_{\mathrm{ir}} 2.1$ channels lower the resting potential to $-70 \mathrm{mV}$, priming the $\mathrm{Na}_{V} 1.5$ channels for activation. Upon depolarization to voltages greater than $-50 \mathrm{mV}$, the sodium channels activate, leading to a voltage spike. The $\mathrm{Na}_{V} 1.5$ channels then inactivate and the $\mathrm{K}_{\mathrm{ir}} 2.1$ channels return the membrane to its resting potential. To provide a bidirectional optical interface, we also stably express a blue light-activated channelrhodopsin, CheRiff [Fig. 1(b)], and a red light-excited fluorescent voltage indicator, QuasAr2
[Fig. 1(c)], linked by a self-cleaving P2A peptide [24] (see the Appendix). The HEK cells endogenously express a low level of gap junctions [25], which provides nearest-neighbor electrical coupling. We call the engineered cell line Optopatch spiking HEK cells (OS-HEK cells).

We characterize the cells via simultaneous manual patch clamp and fluorescence measurements, while stimulating with pulses of blue light of increasing intensity $(488 \mathrm{~nm}$, 6 pulses of $10 \mathrm{~ms}$ duration from 15 to $115 \mathrm{~mW} / \mathrm{cm}^{2}$ ). At blue stimulus intensity $\leq 60 \mathrm{~mW} / \mathrm{cm}^{2}$, cells show small electrical depolarizations with magnitude proportional to illumination intensity. At blue stimulus intensity of $90 \mathrm{~mW} / \mathrm{cm}^{2}$, cells spike to $+33 \mathrm{mV}$, close to the sodium reversal potential, and then gradually return to baseline. Illumination at higher intensities does not lead to further increases in spike amplitude, a defining characteristic of action potentials. The QuasAr2 fluorescence closely follows the electrical recording, with an action potential amplitude of $6 \% \Delta F / F$. These results establish the feasibility of simultaneous optical stimulation and optical readout of electrical spikes in OS-HEK cells.

To probe collective dynamics, we develop a wide-field optical system for simultaneous imaging and stimulation. To achieve high optical sensitivity over a large field of view, we use a large-aperture objective lens (Olympus MVX-2, 2x, NA 0.5), custom extra large dichroic mirrors $(60 \times 85 \mathrm{~mm})$, and a custom imaging path (see the Appendix). Because of the low brightness of QuasAr2, it is necessary to illuminate the sample with high-intensity red light $\left(635 \mathrm{~nm}, 13 \mathrm{~W} / \mathrm{cm}^{2}\right)$ over the whole imaging area $\left(5 \times 1 \mathrm{~mm}^{2}\right)$. To avoid autofluorescence of the glass in the objective lens, the red light is brought into the sample through a custom fused silica prism coupled to the bottom of the sample via immersion oil (see Fig. 1 in Supplemental Material [23] and the Appendix) [24]. The laser enters the sample just below the angle for total internal reflection at the glass-water interface, minimizing illumination of the glass below or the buffer above the cells. Fluorescence from the sample passes through the prism to the objective. Patterned blue illumination is provided by a digital micromirror device (DMD) with a resolution of $912 \times 1140$ pixels. Individual cells within a confluent monolayer $\left(\sim 160000\right.$ cells $\left./ \mathrm{cm}^{2}\right)$ are resolvable via the fluorescence of CheRiff-eGFP [Fig. 1(e)]. The field of view comprises $\sim 8000$ OS-HEK cells. At high cell densities we often observe spontaneous spiral waves of fluorescence propagating through the dish (see Movie 1 in Supplemental Material [23]).

We stimulate the sample with a bar of blue light $\left(\lambda=488 \mathrm{~nm}, 200 \mathrm{~mW} / \mathrm{cm}^{2}, 300 \mu \mathrm{m} \times 1 \mathrm{~mm}, 50 \mathrm{~ms}\right.$ duration) and record a movie of the ensuing changes in QuasAr2 fluorescence. An action potential wave propagates through the syncytium away from the bar at a velocity of $2.3 \mathrm{~cm} / \mathrm{s}$ [Fig. 1(f)], establishing that a confluent layer 
(a)

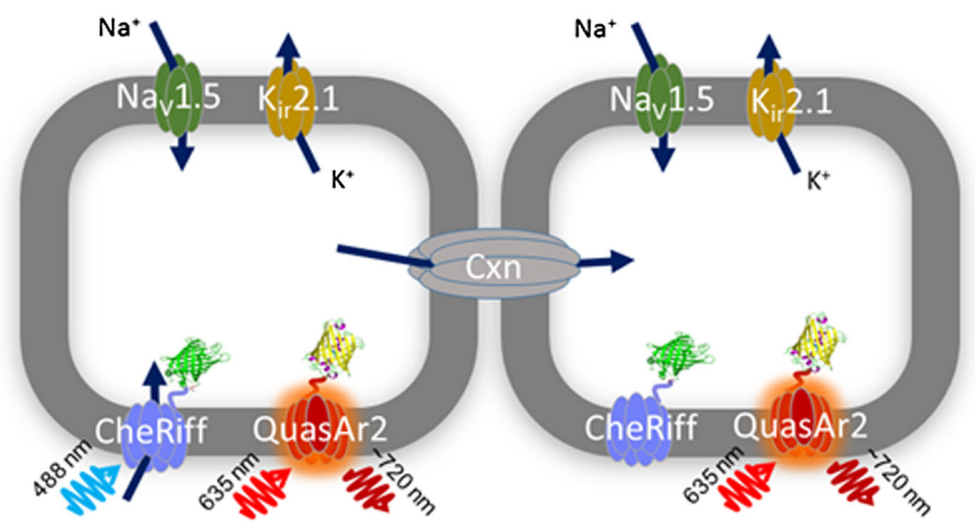

(b)

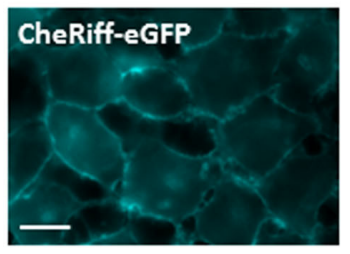

(c)

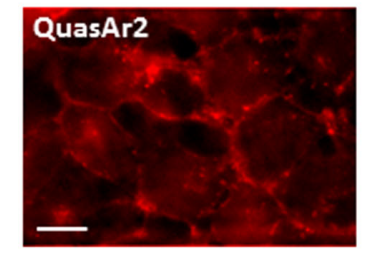

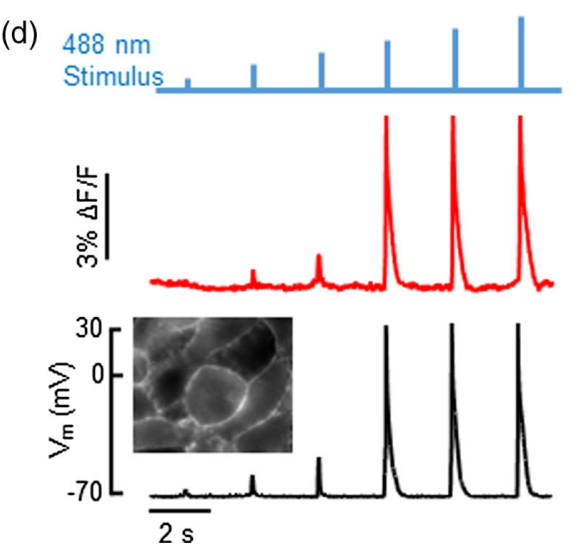

(e)

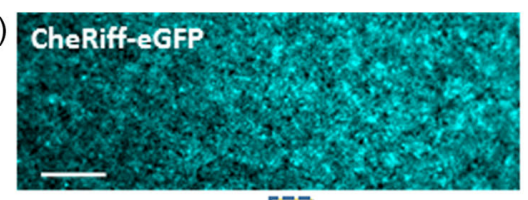

(f)
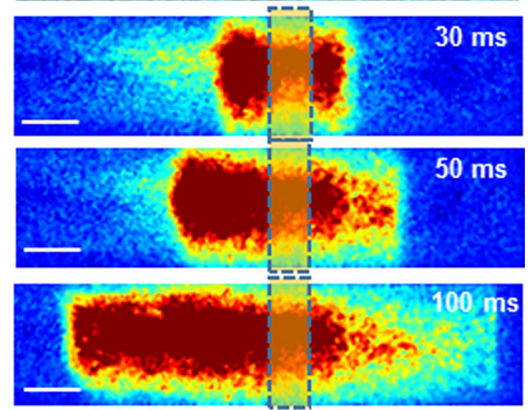

(g)
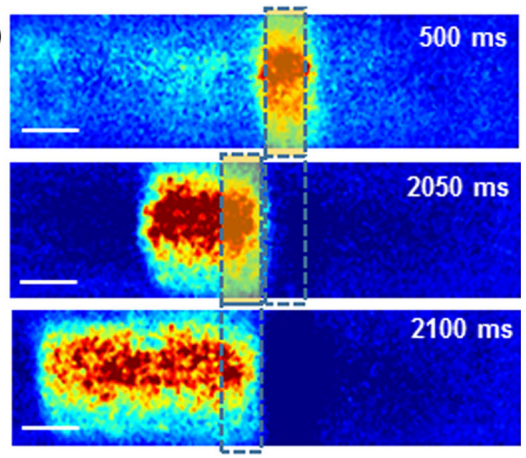

FIG. 1. Optopatch spiking HEK (OS-HEK) cells enable optical stimulation and optical readout of electrical waves in confluent monolayers. (a) Functional components of OS-HEK cells. Two transgenic ion channels $\left(\mathrm{Na}_{V} 1.5, \mathrm{~K}_{\mathrm{ir}} 2.1\right)$ imbue the cells with the ability to produce electrical spikes. Two optogenetic components (CheRiff and QuasAr2) provide optical input and output, respectively. Endogenous gap junctions provide electrical coupling between neighboring cells. (b) Image of eGFP fluorescence in CheRiff-eGFP. (c) Image of QuasAr2 fluorescence. Scale bars in (b) and (c) $20 \mu \mathrm{m}$. (d) Simultaneous fluorescence recording (red, $\lambda_{\text {exc }}=635 \mathrm{~nm}$, $1 \mathrm{~kW} / \mathrm{cm}^{2}, \lambda_{\mathrm{em}}=668-743 \mathrm{~nm}$ ) and patch clamp measurements (black) during ramped optogenetic stimulation (blue). Inset: Image of the recorded cell. (e) Wide-field image of eGFP fluorescence in a confluent layer of OS-HEK cells. (f) Images of QuasAr2 fluorescence showing wave propagation induced by a flash of blue light delivered to the dashed box. (g) Directional waves are launched by inactivating a zone (top) and then stimulating an adjacent region (middle). Scale bars in (e)-(g) $500 \mu \mathrm{m}$. See Movie 2 in Supplemental Material [23].

of OS-HEK cells could support collective wave propagation, with optical input and output.

To launch unidirectional waves, we use the fact that static depolarization leads to inactivation of sodium channels. A bar-shaped region is illuminated with a long-lasting blue pulse $\left(2 \mathrm{~s}, 200 \mathrm{~mW} / \mathrm{cm}^{2}\right)$. An adjacent bar is then illuminated with a brief blue pulse $\left(50 \mathrm{~ms}, 200 \mathrm{~mW} / \mathrm{cm}^{2}\right)$. The resulting wave propagates unidirectionally away from the statically illuminated region [Fig. 1(g) and Movie 2 of Supplemental Material [23]). A similar protocol has been used to launch directional waves in cardiac cell cultures [18].

\section{RING OSCILLATORS AND BIOELECTRIC MEMORIES IN PATTERNED OS-HEK CELLS}

The capability of OS-HEK cells to support optically triggered wave propagation suggested that these cells might be configured as stable oscillators. We use soft lithography [26] to pattern the cell growth into annular patterns with diameters ranging from 1 to $5 \mathrm{~mm}$ [see Fig. 2(a) and the Appendix). We then use the illumination sequence of Fig. 1(g) to launch unidirectional waves in these rings (see Movie 3 in Supplemental Material [23]). Waves propagate stably in rings of diameter 2, 3, and $4 \mathrm{~mm}$, but in $1 \mathrm{~mm}$ diameter rings waves catch their tails and quench.

Waves can be launched clockwise or counterclockwise, stopped with a bar of static illumination, and then relaunched in either direction [Figs. 2(b)-2(d)]. The direction of wave propagation constitutes a topologically stable bit of information. To explore the capabilities of this bioelectric memory, we write all four two-bit patterns of oscillation into two concentric rings. The handedness remains stable for $>2 \mathrm{~h}$, corresponding to $\sim 10^{4}$ oscillation cycles [Fig. 2(e)]. Over $2 \mathrm{~h}$, the oscillation frequency in the 3-mm diameter ring drifts by $4.8 \%$, from 2.37 to $2.25 \mathrm{~Hz}$.

As in any physical oscillator, long-term stability enables us to detect subtle shifts in frequency, in this case arising 
(a)

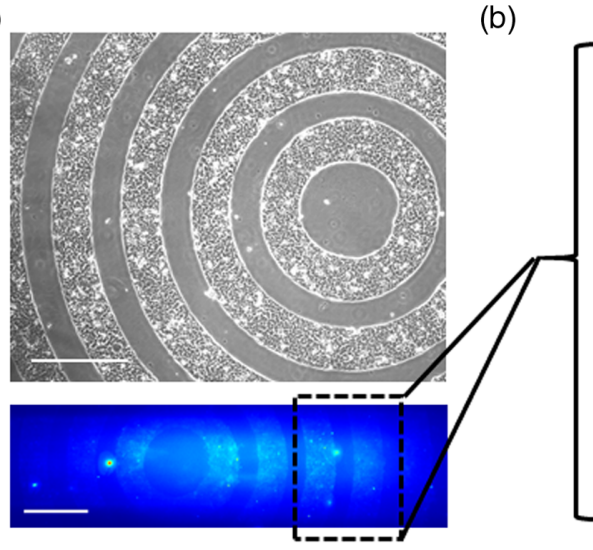

(b)

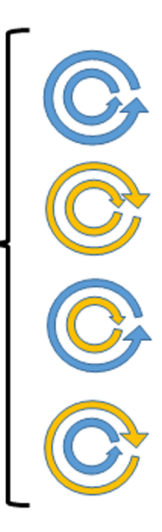

$t=0$
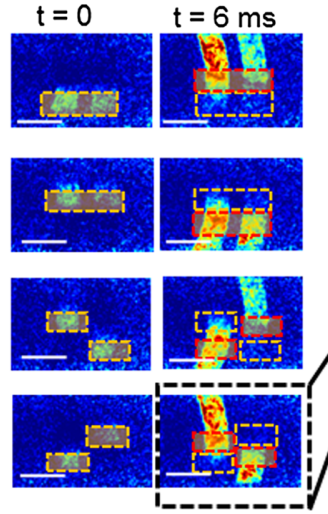

(c)

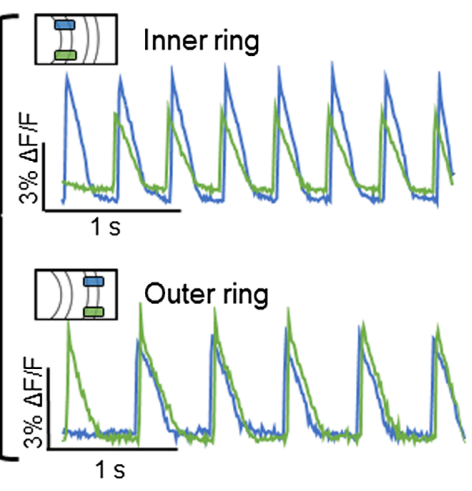

(d)

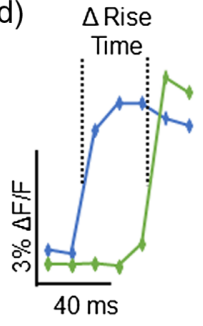

(e)

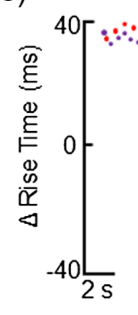

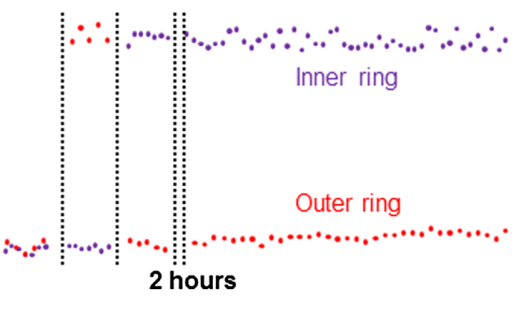

(f)

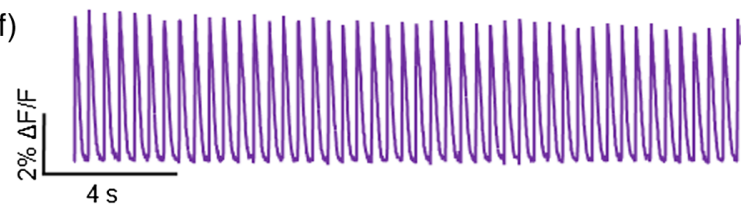

FIG. 2. Ring oscillators in annular OS-HEK cell cultures. (a) Top: Transmitted light image of micropatterned rings of OS-HEK cells. Bottom: The fluorescence field of view is limited to a slice through the rings. (b) Optical initiation of directional waves in the annular cultures. In two concentric rings, waves are launched sequentially in all four binary combinations of directions. Dashed boxes indicate the illumination sequences used to launch the waves. Scale bars in (a) and (b), $1 \mathrm{~mm}$. (c) Intensity patterns of QuasAr2 fluorescence after wave initiation. (d) A slight time shift in the upstroke of the waves measured at different locations on the annulus indicate the direction of circulation. (e) Writing of all four two-bit binary patterns. Red and purple dots indicate the direction of propagation in the outer and inner rings, respectively. The last pattern is left undisturbed at room temperature and continues to circulate for $>2 \mathrm{~h}$. (f) Oscillation patterns in regions of the inner and outer rings after $2 \mathrm{~h}$ of continuous circulation, corresponding to $\sim 10^{4}$ cycles. See Movie 3 in Supplemental Material [23].

from changes in conduction velocity (CV). In principle, conduction velocity could be sensitive to any environmental or pharmacological perturbation that affects the ion channels or metabolism of the cells. Burton and co-workers found that conduction velocity in cultured cardiac myocytes could be increased by whole-field weak optogenetic stimulation [18]. We test whether the same is true for OS-HEK cells. In a ring of diameter $3 \mathrm{~mm}$, oscillations in the absence of blue illumination occur at a frequency of $2.44 \mathrm{~Hz}$ and have a transform-limited spectral linewidth of $0.11 \mathrm{~Hz}$ in a 9-s measurement band. Whole-field illumination with dim blue light brings the cells closer to activation threshold and thereby leads to faster conduction, up to a maximum $14 \%$ increase at $60 \mathrm{~mW} / \mathrm{cm}^{2}$ (See Fig. 2 in Supplemental Material [23]). Illumination above $60 \mathrm{~mW} / \mathrm{cm}^{2}$ quenches the oscillation. Transformlimited changes in oscillation frequency as small as $4.2 \%$ in a 10-s measurement window are readily detectable, suggesting possible biosensor applications for rings of OS-HEK cells.

\section{BIOPHYSICAL CHARACTERIZATION OF OPTOPATCH SPIKING HEK CELLS}

Wave propagation in excitable media is governed by the restitution properties of the individual units [10,27], the dispersion relation of the medium (dependence of conduction velocity on excitation rate) [28], and the effect of curvature on wave front velocity $[29,30]$. These parameters have previously been studied in cardiac tissue [31], though until recently stimulation with physical electrodes imposed constraints on stimulus geometry. Patterned optogenetic stimulation combined with voltage imaging provided a facile measurement platform to characterize in detail these biophysical parameters in cultures of OS-HEK cells.

\section{A. Dispersion relations}

In a periodically stimulated excitable medium, each wave front propagates in the wake of the preceding wave. If the interval between waves is comparable to or shorter than the recovery time of the medium, 
conduction velocity and action potential duration depend on stimulus frequency.

We measure the dispersion relations in a homogenous OS-HEK culture by launching periodic trains of ten waves with a bar-shaped stimulus $\left(50 \mathrm{~ms}, 200 \mathrm{~mW} / \mathrm{cm}^{2}\right.$, $125 \mu \mathrm{m} \times 6 \mathrm{~mm}$ ), with repetition frequency from 0.5 to 4.0 Hz. For each wave, we characterize the conduction velocity [Fig. 3(a)] and action potential duration (AP50, FWHM; see Fig. 3 in Supplemental Material [23]). The spatial extent of the propagating depolarization is then given by $L=\mathrm{AP} 50 \times \mathrm{CV}$. Between trains of different frequencies, the sample is given $2 \mathrm{~s}$ to relax. We observe a weak decrease of conduction velocity with increasing frequency between 0.5 and $2.5 \mathrm{~Hz}$ [Fig. 3(a)], and also a weak decrease of AP50 over this frequency range (see Fig. 3 in Supplemental Material [23]). At stimulus frequencies $>2.5 \mathrm{~Hz}$, it is not possible to quantify wave propagation because the stimulus triggers reentrant spiral waves, which interfere with smooth propagation of optically triggered waves (see Movie 4 in Supplemental Material [23]).

\section{B. Curvature-dependent velocity}

We quantify the effect of wave front curvature $\kappa$ on $C V$ in OS-HEK monolayers by launching either circular outgoing waves ( $\kappa>0$, Movie 5 of Ref. [23]) or annular incoming waves $[\kappa<0$, Fig. 3(b)]. High-speed $(500 \mathrm{~Hz})$ maps of the wave fronts reveal that $\mathrm{CV}$ depend sensitively on $\kappa$ [Fig. 3(c)]. For a planar wave front to be stable, CV must be a monotonically decreasing function of $\kappa$, consistent with our observations. We compare our observations to numerical simulations of the Aliev-Panfilov model [32], a simple two-variable description of an excitable medium (see the Appendix). The simulations reproduce the qualitative features of the data, including an asymmetry between positive and negative curvatures. Because of the simplicity of the Aliev-Panfilov model, precise agreement with experiment is not expected.

The liminal radius is the smallest stimulus radius that can initiate an outward-propagating wave [33]. Applying a linear extrapolation of the positive-curvature data to $\mathrm{CV}=0$ yields a liminal radius of $25 \mu \mathrm{m}$. Optically excited (a)

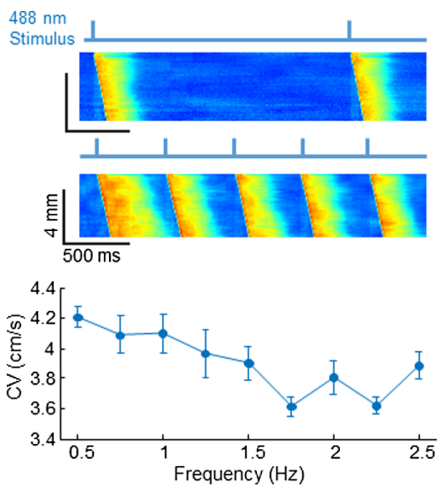

(b)

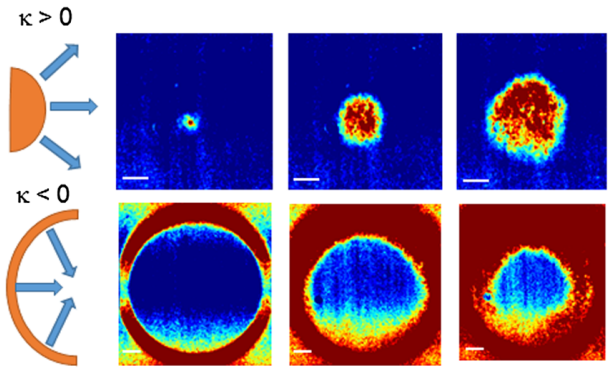

(c)

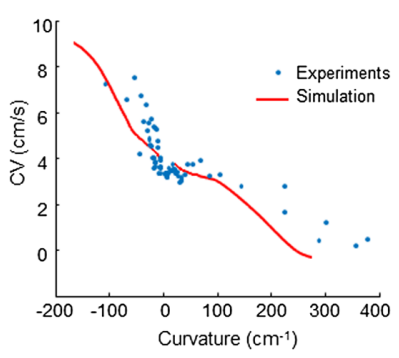

(d)

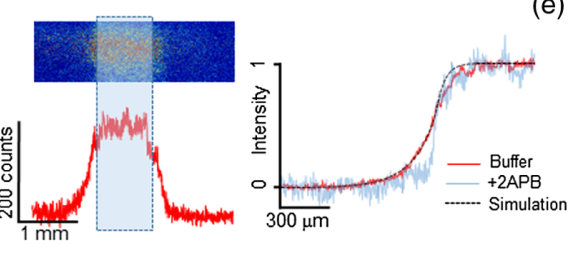

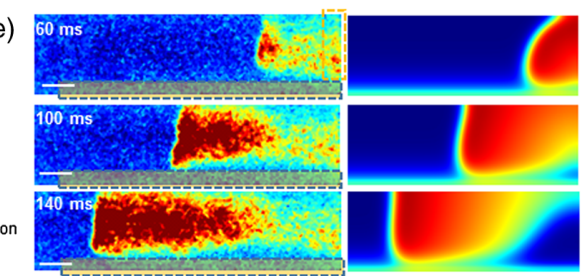

FIG. 3. Wave propagation in OS-HEK cell cultures. (a) Dispersion relation. Samples are stimulated with a vertical bar of blue light for $50 \mathrm{~ms}, 10$ times in succession, with variable frequency. Movies of fluorescence are converted into kymographs by averaging parallel to the wave front. Top: Example kymographs recorded at 0.5 and $2 \mathrm{~Hz}$. Bottom: Mean conduction velocity as a function of stimulus frequency. Error bars represent standard deviation of individual wave measurements within a train. (b) Curvature-dependent conduction velocity. Waves are launched either outward from a small spot (top) or inward from an annular ring (bottom) to probe positive and negative curvatures, respectively. Scale bars $500 \mu \mathrm{m}$. Successive panels are separated by $10 \mathrm{~ms}$. (c) Scatter plot of conduction velocities as a function of curvature (blue points). Each point represents a single frame of a particular wave. Data are compiled across several trials with differing fields of view to accommodate both low and high curvatures ( $n=10$ trials). Red: numerical simulations based upon Aliev-Panfilov model. (d) Static electrotonic coupling. Top: Cells are illuminated with a bar of blue illumination of sufficient intensity $\left(200 \mathrm{~mW} / \mathrm{cm}^{2}\right)$ to saturate CheRiff photocurrent. Bottom: Mean QuasAr2 intensity profile transverse to the bar of illumination. The CheRiff-induced depolarization spread laterally away from the illuminated zone. Right: Comparison of measured depolarization profile to a numerical fit based on the Aliev-Panfilov model with a coupling length of $300 \mu \mathrm{m}$. A gap junction blocker (2-aminoethoxydiphenyl borate) suppresses the lateral spread of depolarization. (e) Static illumination guides to propagation. Left: A wave is launched adjacent to a region of static illumination (indicated with dotted box on the bottom of the images). The wave propagates parallel to the boundary, with local inhibition adjacent to the boundary. Right: Comparison to simulations of the Aliev-Panfilov model. Scale bars $500 \mu \mathrm{m}$. See Movies 7 and 8 in Supplemental Material [23]. 
regions smaller than this radius are predicted to be unstable to spontaneous collapse, while larger regions are predicted to trigger an outward propagating wave. Consistent with this extrapolation, we experimentally observe that stimuli smaller than $25 \mu \mathrm{m}$ in radius fail to trigger outward waves, although large local variations in cell density on this size scale lead to considerable region-to-region variation. The liminal size is of clinical interest, as it relates to the sensitivity of the myocardium to ectopic depolarization. Previous work has investigated this parameter in cardiac tissue by varying the diameter of the stimulating electrode [33] or optical fiber for optogenetic stimulation [34].

\section{Optically controlled boundary conditions}

We now explore the feasibility of using static illumination patterns to create inexcitable zones for the purpose of steering optically induced waves. Movie 6 of the Supplemental Material [23] shows that static illumination of a bar-shaped region creates a barrier for propagating waves. We interpret this local loss of excitability as a consequence of depolarization-induced inactivation of $\mathrm{Na}_{V}$ channels.

Since we seek to form patterns of high complexity, we next study the spatial resolution with which we can optically modulate membrane potential. Static illumination of a bar-shaped region leads to a steady-state depolarization that decays to half its maximum at $160 \mu \mathrm{m}$ beyond the edge of the illumination [Fig. 3(d)]. To verify that the observed broadening is due to electrical coupling (as opposed to optical scattering or imaging aberrations), we add a gap junction blocker, 2-aminoethoxydiphenyl borate (2-APB, $5 \mu \mathrm{M})$. This drug prevents wave propagation and leads to a steplike decrease in fluorescence at the edge of the static illuminated region [Fig. 3(d)], confirming that the spread in fluorescence beyond the illuminated region is due to electrotonic coupling.

We compare our results to numerical simulations of the Aliev-Panfilov model [32]. We determine the cell-cell coupling parameter in the model by fitting to the data on electrotonic spread of static depolarization [Fig. 3(d); see also the Appendix]. This fit gives a dimensionless coupling length of $\phi=12.5$, corresponding to $\phi=300 \mu \mathrm{m}$ in our experiments. This parameter sets the spatial resolution with which patterns of static illumination can modulate the potential.

One can imagine that the partially depolarized region adjacent to the static illumination could act as a pacemaker, shedding periodic waves into the unstimulated region. We find this not to be the case. No degree of static blue light stimulus induces periodic activity. We speculate that the shift of the $\mathrm{Na}_{V} 1.5$ population to the slow inactivated state suppresses oscillations for any degree of static depolarization [35].
We next use patterns of static illumination to guide wave propagation. We illuminate a bar of cells with static blue light, and then use a pulse of blue light to launch a wave propagating along this optical boundary. We observe a convex curved wave front near the static interface (Movies 7 and 8 of Supplemental Material [23]). The wave front is retarded immediately adjacent to the boundary, but then slightly advances further out. As the wave propagates, the advanced region gradually spreads outward from the boundary, until eventually the wave appears to have a straight wave front outside the boundary region.

We ascribe the nonmonotonic dependence of conduction velocity on distance from a depolarized boundary to the nonmonotonic dependence of sodium channel activation on membrane voltage [36]. Large depolarizations (close to the optical boundary) inactivate sodium channels and hence slow conduction. Modest depolarizations (farther from the boundary) bring the sodium channels closer to the activation threshold and hence speed conduction. This qualitative picture is consistent with our prior observations that weak whole-field blue illumination accelerates wave propagation, while strong blue illumination suppresses wave propagation.

We observe close correspondence to numerical simulations of the Aliev-Panfilov model using the coupling length determined by fit to the static depolarization profile. We reproduce propagation patterns both along a depolarized boundary [Fig. 3(e)] and around a corner (see Fig. 4 and Movie 8 in Supplemental Material [23]). We find that, due to the spread of inactivation near optical boundaries, optically defined channels have to be at least $\sim 300 \mu \mathrm{m}$ wide to support wave conduction.

\section{OPTICALLY DEFINED RING OSCILLATOR}

Finally, we seek to define a cellular circuit entirely optically. In contrast to physically patterned circuits, patterned illumination can be used to dynamically reconfigure circuit boundaries, enabling new types of circuit function.

Starting with a homogeneous confluent culture of OS-HEK cells, we illuminate with static blue light to define the boundaries of a rectangular track [Fig. 4(a)]. We then optically launch a counterclockwise wave within the track. The wave circulates stably within the optically defined boundaries at constant frequency. We then dynamically change the dimensions of the track, and observe a corresponding change in the oscillation frequency (see Movie 9 of Ref. [23]). Upon removing the optical boundaries, the wave expands outward and dissipates at the edges of the dish. The spectrogram of the intensity trace demonstrates the expected increase of oscillator velocity as the circumference shrinks [Fig. 4(c)]. These results demonstrate that one can use optical patterning to define dynamically reconfigurable boundary conditions in cultures of OS-HEK cells. 
(a)

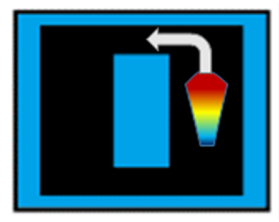

(b)
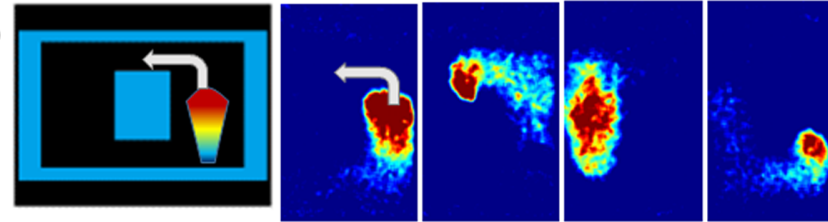

(c)

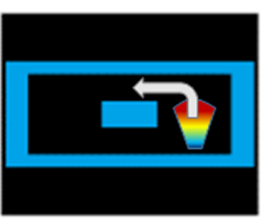

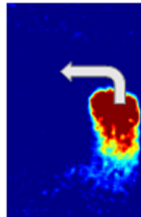
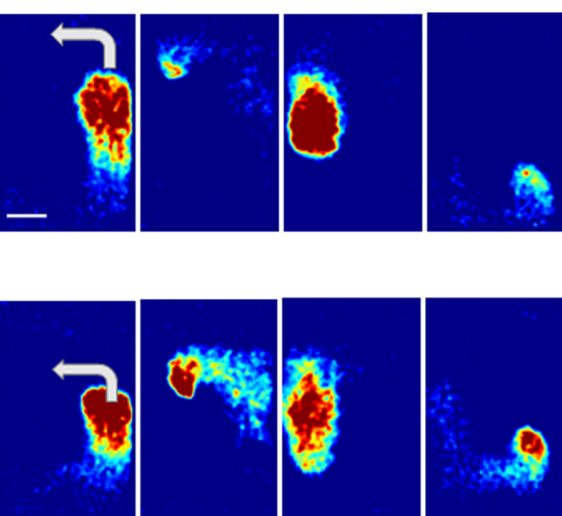

a
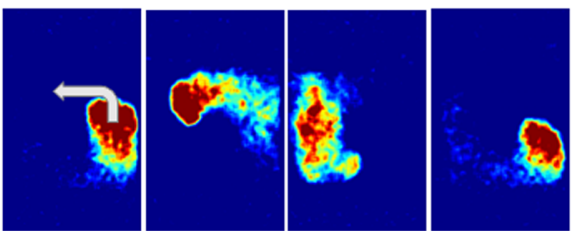

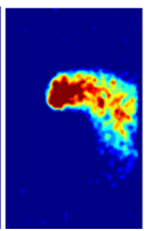

(d)

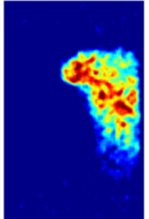

(e)

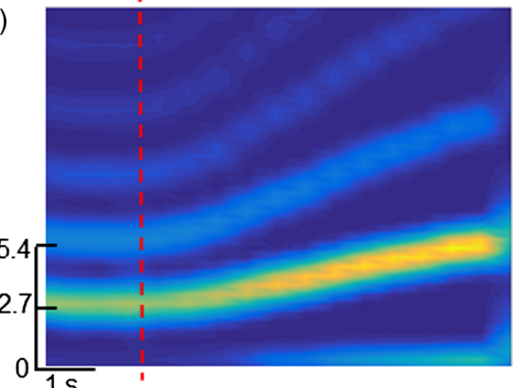

FIG. 4. Dynamic optical tuning of OS-HEK cell oscillators. (a)-(c) Circular wave motion in an optically defined rectangular track, defined by the pattern of blue illumination shown on the left. The boundaries are applied as a quasistatic illumination pattern, and then a directional wave is launched to initiate counterclockwise circulation. The top and bottom optical boundaries are then continuously shrunk during the recording. The apparent decrease in signal amplitude at the top and bottom of the largest pattern arises because the waves passed the edge of the fluorescence excitation spot. Scale bars $1 \mathrm{~mm}$. Movies acquired at $100 \mathrm{~Hz}$, successive panels separated by 8-10 frames. (d) QuasAr2 fluorescence from the indicated region. As the vertical dimension of the track shrinks, the oscillations speed up. (e) Spectrogram of the data in (d) showing the increase in frequency with time. See Movie 9 in Supplemental Material [23].

\section{DISCUSSION}

OS-HEK cells and the associated optical instrumentation provide a versatile platform for exploring emergent bioelectric phenomena in excitable tissues. The two heterologously expressed ion channels, $\mathrm{Na}_{V} 1.5$ and $\mathrm{K}_{\mathrm{ir}} 2.1$, combined with the endogenous gap junction protein, constitute a minimal system for sustaining collective electrical waves in vitro. The ability to define boundary conditions optically and to vary these conditions in real time opens the possibility to explore the effects of geometry on wave propagation phenomena with much greater precision and versatility than would be possible if physical cell patterning were required. For instance, measurements of conduction velocity versus wave front curvature [Fig. 3(c)] reveal a nonlinear dependence, indicating deviations from the linear eikonal approximation [37]. A similarly shaped dependence of velocity on wave front curvature was previously observed in calcium waves within single isolated rat cardiomyocytes [30]. To our knowledge, our results constitute the most precise measurement of the influence of wave front geometry on electrical conduction in a biological tissue.

More than 100 years ago, Mines considered rings of cardiac tissue as a model for reentrant arrhythmias and observed stabilization of reentrant waves at high stimulus rates [38]. More recent work has considered annular geometries in cardiac electrical conduction $[39,40]$, in cardiac calcium dynamics [41], and in chemical reactiondiffusion systems [42]. In contrast to previous work in fixed geometries, our optical approach opens the door to exploring how reconfiguration of boundary conditions affects dynamics in a bioelectric medium. In principle, one could close the loop between optical readout of voltage and optical stimulation to provide spatiotemporal feedback in OS-HEK cells, an idea first discussed by Sakurai et al. [43].

Spiking HEK cells have previously been proposed as a model of cardiac tissue [16]. The quantitative data obtained here test the limits of this model and reveal instructive differences from real cardiac tissue. The relations of conduction velocity and AP50 to beat rate determine the stability of wave propagation in the tissue $[44,45]$. If the slope of the restitution curve (AP50 versus diastolic interval) exceeds 1 , then under periodic pacing electrical alternans (alternating short and long beats) develops [46], a phenomenon first characterized by Mines [47]. Guevara and co-workers showed that this transition can occur via a period-doubling bifurcation, and can eventually lead to chaotic dynamics $[10,27]$. Transitions to chaos may be involved in ventricular fibrillation and sudden cardiac death [48].

In OS-HEK cells the conduction velocity and AP50 are largely independent of stimulus frequency (and hence diastolic interval), up to a sharp cutoff frequency [Fig. 3(a) and Fig. 3 in Supplemental Material [23]]. Neither 
alternans nor chaos are observed in the periodically paced OS-HEK cells, the physically patterned rings of OS-HEK cells, or in the optically defined ring oscillator. To interpret this qualitative difference from cardiac tissue, we turn to the classic Noble model [49], in which cardiac dynamics are described by three currents: voltage-gated sodium, inward rectifier potassium, and slow delayed rectifier potassium. OS-HEK cells lack the slow delayed rectifier potassium channel of the Noble model, and thus recover after each action potential within a time set by sodium channel repriming, approximately $4 \mathrm{~ms}$ [50]. Thus, each beat in OS-HEK cells is largely independent of preceding activity. The lack of the slow delayed rectifier current in OS-HEK cells most likely explains the difference in dispersion relation between OS-HEK cells and cardiac tissue.

One could augment the OS-HEK cells in several ways to make them more "cardiaclike." Expression of a slow $\mathrm{K}_{V}$ channel, such as hERG, would provide a gradually recovering excitability after each action potential. A slow hyperpolarization-activated cation channel, such as an HCN channel, would lead to a gradual depolarizing "funny current" after each action potential, also gradually increasing excitability [51]. Finally, one could add a drug, such as amitriptyline, which slows the recovery of $\mathrm{Na}_{V} 1.5$ from inactivation and may thus lead to slowly recovering excitability [52]. The OS-HEK system provides a platform for testing the sufficiency of these additional components to induce alternans and chaotic dynamics under rapid pacing. It will also be interesting to explore whether these modifications render the cells capable of driving oscillatory behavior under steady-state optogenetic stimulation.

One could also augment the OS-HEK cells to implement more complex cellular logic. For instance, expression of A-type voltage-gated potassium channels should shorten the AP duration to resemble more closely a neuronal action potential. The correspondingly shorter AP wavelength would lead to a higher bit rate in propagating wave trains. Expression of voltage-gated $\mathrm{Ca}^{2+}$ channels may introduce additional slow dynamics and memory effects, and provide a means to couple electrical dynamics to intracellular signaling cascades and ultimately to changes in gene expression.

Expression of ligand-gated ion channels could provide a sensitive chemical interface, whose activation would manifest as changes in wave dynamics. Activation of G-protein coupled receptors (GPCRs) can also couple to ion channel modulation [53] and thereby affect the function of OS-HEK cells. The ring oscillator geometry enables integration over thousands of oscillation cycles, facilitating detection of extremely subtle changes in oscillation frequency. Finally, patterned cell growth of multiple engineered cell types in two and three dimensions [54] may enable formation of highly complex bioelectrically active synthetic tissues, with cells having differing electrophysiological properties localized in different regions. An interesting avenue for future research will be to engineer cell lines to perform more complex bioelectrical computations.

\section{ACKNOWLEDGMENTS}

We thank Miao-Ping Chien for help with cell patterning and Katherine Williams and Melinda Lee for technical support. This work was supported by ONR Grant No. N000141110-549, NIH Grants No. 1-R01-EB01249801 and New Innovator Grant No. 1-DP2-OD007428, and the Howard Hughes Medical Institute. H. M. M. designed, conducted, and analyzed experiments. H.Z. created the OS-HEK cell line. C. A. W. created the wide-field imaging system. A. E. C. oversaw the research. H. M. M. and A. E. C. wrote the paper.

\section{APPENDIX: MATERIALS AND METHODS}

\section{Generation of OS-HEK cell lines}

The pIRESpuro3-Na $\mathrm{Na}_{V} 1.5$ plasmid was obtained from ChemCORE at Johns Hopkins University. The $\mathrm{Na}_{V} 1.5$ expression is driven by a CMV promoter. The Optopatch construct contains coding sequences of CheRiff-eGFP and QuasAr2-mOrange, separated by a self-cleaving P2A peptide sequence. The Optopatch construct was cloned into a modified FCK lentivirus vector (mFCK), in which the original CaMKII promoter was replaced by a CMV promoter. The $\mathrm{K}_{\mathrm{ir}} 2.1$ cDNA is amplified from Addgene plasmid 32669 (pENTR-L5-Kir2.1-mCherry-L2) and cloned into pLX304 lentivirus vector that contains a blasticidin selection marker. The $\mathrm{K}_{\mathrm{ir}} 2.1$ expression is driven by a CMV promoter.

HEK293 cells are maintained in Dulbecco's modified Eagle medium (DMEM) with 10\% fetal bovine serum, penicillin $(100 \mathrm{U} / \mathrm{mL})$, streptomycin $(100 \mu \mathrm{g} / \mathrm{mL})$, and are transected with pIRESpuro3-Na 1.5 using TransIT-293 transfection reagent (Mirus Bio) following manufacturer instructions. $48 \mathrm{~h}$ after transfection, puromycin was added to a final concentration of $2 \mu \mathrm{g} / \mathrm{mL}$. Cells are selected for 14 days to stabilize the expression of $\mathrm{Na}_{V} 1.5$. Surviving cells are subsequently transduced by mFCK-Optopatch lentivirus. Ten days after infection, GFP positive cells are enriched by FACS sorting and are then transduced by pLX304-Kir2.1 lentivirus. $48 \mathrm{~h}$ after transduction, $\mathrm{K}_{\mathrm{ir}} 2.1$ expressing cells are selected by $5 \mu \mathrm{g} / \mathrm{mL}$ blasticidin. At the same time, $2 \mu \mathrm{g} / \mathrm{mL}$ puromycin is also included to ensure stable expression of $\mathrm{Na}_{V} 1.5$. Cells are cultured for 14 days and then single cells are dispersed in wells of a 48-well plate. Monoclonal lines are screened using Optopatch to detect cells that show robust optically induced and optically recorded action potentials. In the selected $\mathrm{Na}_{V} 1.5 /$ Optopatch/K $\mathrm{K}_{\text {ir }} 2.1$ monoclonal cell line (OS-HEK cells), action potentials are robustly triggered by blue laser stimulation and recorded by QuasAr2 fluorescence, with a SNR greater than 30 . 


\section{Cell culture}

The selected OS-HEK cell line is grown in 10-cm tissue culture dishes in 10-mL DMEM-10 supplemented with $2 \mu \mathrm{g} / \mathrm{mL}$ puromycin and $5 \mu \mathrm{g} / \mathrm{mL}$ blasticidin until reaching approximately $80 \%$ confluence. Cells are then were trypsinized and transferred to cryovials with 500000 cells/vial in $10 \%$ dimethyl sulfoxide (DMSO)/ 90\% DMEM-10 medium, and frozen overnight at $-80^{\circ} \mathrm{C}$ using an isopropyl alcohol freezing container (Thermo Scientific).

For functional experiments, OS-HEK cells are thawed and centrifuged at $300 \mathrm{~g}$ for $5 \mathrm{~min}$ to pellet cells and aspirate DMSO-containing medium. Cells are then resuspended and plated in 10-cm plastic tissue culture dishes in 10-mL DMEM-10 supplemented with $2 \mu \mathrm{g} / \mathrm{mL}$ puromycin and $5 \mu \mathrm{g} / \mathrm{mL}$ blasticidin. Following standard HEK cell culture convention, OS-HEK lines are maintained between $30 \%$ and $70 \%$ confluence, and are passaged (by trypsinizing, centrifuging, aspirating old media, and resuspending in fresh media) at a 1:3 ratio once reaching $70 \%$ confluence. After thawing, a given line typically maintains functional performance (i.e., spiking under blue light stimulation) for approximately seven passages, or 2 weeks. At longer times in culture, expression of $\mathrm{K}_{\mathrm{ir}} 2.1$ appears to diminish, rendering the cells inexcitable.

For imaging and electrophysiology experiments, cells are passaged from $10-\mathrm{cm}$ plastic dishes and replated on 35-mm coverslip-bottom dishes that have been precoated with 1:30 matrigel:minimal essential media (MEM) in a central $10-\mathrm{mm}$ well to allow for cell adhesion. For confluent layers, cells are plated at high density by delivering 500000 cells in a 500- $\mu$ L DMEM-10 droplet in the central well, then left to settle for $1 \mathrm{~h}$ before adding an additional $1.5 \mathrm{~mL}$ DMEM-10 to the dish. Cells are then transferred to the incubator for at least $3 \mathrm{~h}$ before measurement.

\section{Electrophysiology and single-cell imaging}

Measurements are performed in Tyrode's solution, containing (in mM) $125 \mathrm{NaCl}, 2 \mathrm{KCl}, 2 \mathrm{CaCl}_{2}, 1 \mathrm{MgCl}_{2}, 10$ HEPES, 30 glucose. The $p \mathrm{H}$ is adjusted to 7.3 with $\mathrm{NaOH}$ and the osmolality is adjusted to 305-310 mOsm with sucrose. Prior to measurements, 35-mm dishes are washed twice with $1-\mathrm{mL}$ phosphate-buffered saline (PBS) to remove residual culture media, then filled with $2 \mathrm{~mL}$ Tyrode's solution. Filamented glass micropipettes (WPI) are pulled to a resistance of 5-10 $\mathrm{M} \Omega$ and filled with internal solution containing (in $\mathrm{mM}$ ) $140 \mathrm{KCl}, 1 \mathrm{MgCl}_{2}$, 10 EGTA, 10 HEPES, $3 \mathrm{Mg}$-ATP, $p \mathrm{H}$ adjusted to 7.3 with $\mathrm{KOH}$.

Simultaneous patch clamp and QuasAr2 imaging experiments are performed on a home-built inverted microscope using a low-noise patch clamp amplifier (A-M Systems, model 2400). Voltage traces are collected under $i=0$ current clamp mode. Blue light for optical stimulation (Coherent Obis) is modulated using an acousto-optic tunable filter (Gooch and Housego GH18A series). Red light for monitoring QuasAr2 fluorescence (Dragon Lasers M series, $635 \mathrm{~nm}$ ) is modulated independently using a shutter. Fluorescence in the near infrared is filtered using a dichroic mirror (quadband 405/488/532/635) and an emission filter (668-743 nm) and recorded using a scientific complementary metal-oxide semiconductor (CMOS) camera (Hamamatsu Orca Flash 4.2). Experiments are performed at ambient room temperature $\left(25^{\circ} \mathrm{C}\right)$.

\section{Ultrawide-field Imaging and microscopy}

Ultrawide-field imaging experiments are performed on a home-built inverted microscope modified from a design described previously [24]. In brief, this system provides a field of view of $5 \times 5 \mathrm{~mm}^{2}$, spatial resolution of $3 \mu \mathrm{m}$, and maximum frame rate of $1 \mathrm{kHz}$. Blue light for CheRiff excitation is produced by a 488-nm laser with $3-\mathrm{W}$ maximum power (Coherent Genesis MX) set to $1-\mathrm{W}$ output. Excitation intensity is modulated using an acousto-optic modulator (NEOS Technologies, AOBD Model N45030-5-6.5) and spatially patterned using a digital micromirror device (DMD) (DLi4130, Digital Light innovations). Physical DMD pixels of linear dimension $6.5 \mu \mathrm{m}$ are demagnified $2 \mathrm{x}$ onto the sample plane for $3.25 \mu \mathrm{m}$ resolution.

Red light for exciting QuasAr2 fluorescence is provided by six 500-mW lasers (Dragon Lasers M series, $635 \mathrm{~nm}$ ). The lasers are first combined pairwise via half-wave plates and polarizing beam splitters. The three resulting beams are then directed onto the sample via a custom fused silica prism interposed between the sample and the objective. The prism directs the beams to the sample at an angle close to total internal reflection at the glass-water interface, achieving an illumination intensity of $13 \mathrm{~W} / \mathrm{cm}^{2}$ at the sample. The red illumination covers a field of view of $5 \times 1 \mathrm{~mm}$. The size of the field of view is limited by available laser power and by concern over laser-induced heating of the sample for larger illuminated regions. Near-IR fluorescence emission is filtered using emission filters (Chroma ET665lp and Semrock quadband 336/510/581/703) and reimaged onto a sCMOS camera (Hamamatsu Orca Flash 4.2).

To achieve accurate stimulation of user-selected regions on the sample, it is necessary to map DMD pixels onto camera pixels. First, a triangular test pattern is projected onto a spatially uniform fluorescence test target, and imaged on the camera. Then, an affine transformation matrix is produced to map DMD pixels to camera pixels. Desired blue stimulation patterns are then manually defined on images of the sample, and transformed back into DMD pixels using the registration matrix. Stacks of illumination pattern images are then preloaded into the DMD volatile memory. Custom LabView software provides trigger signals to synchronize modulation of the red and blue lasers, activation of patterns on the DMD, and camera triggers. 


\section{Cell patterning}

To create physically defined ring oscillators (Fig. 2), we adapt a published microcontact printing protocol [26] to define fibronectin patterns covalently bound to cytophobic polyacrylamide gels. In brief, patterns are designed in AutoCAD and printed on a Mylar transparency (CAD Art Services). The pattern is transferred to a layer of SU-8 3025 on a $\mathrm{Si}$ wafer via contact photolithography, followed by removal of unexposed photoresist with SU-8 developer. The SU-8 master is then used as a template for casting a poly(dimethylsiloxane) (PDMS) stamp.

Coverslip-bottomed 35-mm dishes with 20-mm central wells (MatTek P35G-1.5-20-C.s) are coated with a layer of polyacrylamide gel functionalized with succinimide esters for covalent immobilization of patterned fibronectin. Glass coverslips are chemically activated by plasma cleaning and then incubated for $30 \mathrm{~min}$ in a nitrogen-purged glovebox with silane solution (v/v: 0.5\% 3-methacryloxypropyltrimethoxysilane, $2 \%$ acetic acid, $97.5 \%$ anhydrous $\mathrm{EtOH})$. Still in a nitrogen atmosphere, polyacrylamide gels (40:1 acrylamide:bisacrylamide) are polymerized on the activated glass (final $\mathrm{W} / \mathrm{V}$ concentrations in $p \mathrm{H} 7$ phosphate buffer: $8 \%$ acrylamide, $0.2 \%$ bisacrylamide, $0.1 \%$ tetramethylenediamine, $0.12 \%$ potassium persulfate; $1 \mathrm{~mL} /$ dish). The solution is doped with $4.2 \mathrm{mg} / \mathrm{mL}$ $\mathrm{N}$-acryloxysuccinimide (acryl-NHS) to allow for covalent binding of fibronectin. Gels are polymerized under siliconized coverslips (Hampton Research, HR3-239) to ensure smooth top surfaces.

PDMS stamps are exposed to a solution of fibronectin (Yo Proteins no. 663, $0.05 \mathrm{mg} / \mathrm{mL}$ ) for $30 \mathrm{~min}$ followed by aspiration and drying in air for $10 \mathrm{~min}$. Stamp patterns are then printed onto the NHS-functionalized acrylamide dishes. The NHS is covalently bonded to the fibronectin, forming a stable cell-adherent pattern. Cells are deposited by gently pipetting a $500-\mu \mathrm{L}$ DMEM-10 droplet with 500000 cells into the dish central well, leaving to settle at room temperature under a laminar flow hood for $>30 \mathrm{~min}$. Unadhered cells are removed by gently aspirating the $500-\mu \mathrm{L}$ droplet, then gently adding $2 \mathrm{~mL}$ DMEM- 10 . This protocol yields confluent patterns with high fidelity to the original template [Fig. 2(a)].

\section{Aliev-Panfilov simulations}

We simulate wave propagation in the OS-HEK cell cultures via the Aliev-Panfilov model [32]. The AlievPanfilov model treats the cell as a two-parameter nonlinear oscillator:

$$
\begin{aligned}
& \frac{d u}{d t}=k u(1-u)(u-a)-u v+c+\phi \nabla^{2} u, \\
& \frac{d v}{d t}=\varepsilon(u)(k u-v),
\end{aligned}
$$

where $u$ is the external (i.e., excitation) parameter and $v$ is in the internal (i.e., recovery) parameter. Numerical integration is performed in MATLAB using the ode 45 differential equation solver. To match simulations to single-cell spike data, we choose excitation threshold $a=0.05$, transmembrane current magnitude $k=8$, and recovery factor $\varepsilon(u<a)=1, \varepsilon(u \geq a)=0.03$. The parameter $c$ corresponds to the optical drive, and is a function of space and of time (set to 1 when pixels are illuminated and to 0 when they are not). The parameter $\phi=12.5$ corresponds to the electrical coupling length of the syncytium, and is calibrated by matching the decay length for static depolarization. Nearest-neighbor electrical coupling is implemented using the MATLAB imfilter function with a discrete Laplacian filter and replicating boundary conditions. Display images correspond to simulations of $u(x, y)$ on $128 \times 32$ or $128 \times 45$ grids of Aliev-Panfilov cells run over 4000-85000 time steps, depending the complexity of the simulated optical stimulus.

Simulations of curvature-dependent conduction velocity [Fig. 3(c)] are performed on a $128 \times 128$ grid. Wave fronts are determined by the positions where the intensity crosses a threshold set to $3 / 4$ of the maximum intensity. Trajectories are measured from a one-dimension slice taken across the grid midline, and the spatially discrete results are fit using the MATLAB fit function ("smoothingspline" fit type, with smoothing parameter 0.8). Plots of wave front radius as a function of time are transformed into plots of conduction velocity as a function of curvature.

\section{Image processing and data analysis}

All images are processed and analyzed using homemade MATLAB scripts. For single-cell experiments (Fig. 1), intensity traces are extracted by constructing a weight image based on the covariance of individual pixels with the recorded patch voltage, then using that image to weight contribution of individual pixels to the overall average intensity trace. This algorithm is described in detail in Ref. [55].

For ultrawide-field experiments (Figs. 2-4), movies are background subtracted using an image of the red illumination beam profile and smoothed using a median filter (using a $3 \times 3$ pixel window) to preserve wave front edges. Resulting movies are corrected for photobleaching at each pixel using a sliding minimum filter with a width of 150 samples. This procedure preserves spikes and spike shapes, but removes downward baseline drift. Wave fronts are defined by thresholding movies to identify the wave front and then manually recording the wave front coordinates. Some movies acquired over a large field of view are downsampled using $4 \times 4$ pixel binning to maintain reasonable file size.

For dispersion analysis [Fig. 3(a)], movies are binned at $4 \times 4$ pixels and then averaged over the vertical direction (i.e., parallel to the wave front). The resulting kymographs 
are corrected for photobleaching as described above at each spatial position. The amplitude of the spikes at each coordinate is mapped to the range $[0,1]$, thereby removing variable offsets and amplitudes due to heterogeneities in the culture. Rising and falling edges of each spike are detected using a half-maximum threshold followed by linear interpolation to achieve subframe time resolution.

Conduction velocities are calculated by measuring arrival time at two spatial locations $2 \mathrm{~mm}$ apart. Action potential FWHM (AP50) is calculated by temporally aligning APs at each spatial location, averaging, and then fitting rising and falling times using linear interpolation. For analysis of curvature-dependent velocity [Fig. 3(b)], movies are mean subtracted and then smoothed using a $3 \times 3$ median filter, and then manually traced in ImageJ to determine wave front diameter along the horizontal axis. Velocities are calculated by spline interpolation of the position of the wave front as a function of time, and then taking the spline derivative at sample time points. Associated curvatures are taken as the inverse of the wave front radius measured at that frame. Curvature-dependent velocity data are divided into piecewise linear regimes and then fit with straight lines using least-squares regression to determine the parameter $\alpha$.

For analysis of propagation in the ring oscillator (Fig. 2), raw data are binned, corrected for the red laser illumination profile, and median filtered as described above. Intensity traces are computed from mean intensity within manually defined regions of interest. Fluorescence traces are corrected for photobleaching using a sliding minimum filter as described above. Spike rise times are detected with subframe precision using linear interpolation of samples before and after a half-maximum threshold [Fig. 2(d)]. Oscillator direction is determined by computing differences in rise times at adjacent regions of interest [Fig. 2(e)]. For the optically defined oscillator track (Fig. 4), intensity traces are calculated from a horizontal region in which the boundary did not move during the experiment [Fig. 4(c)]. Power spectra [Fig. 4(d)] are computed by taking shorttime Fourier transforms of these intensity traces.

[1] P. E. Purnick and R. Weiss, The Second Wave of Synthetic Biology: From Modules to Systems, Nat. Rev. Mol. Cell Biol. 10, 410 (2009).

[2] M. B. Elowitz and S. Leibler, A Synthetic Oscillatory Network of Transcriptional Regulators, Nature (London) 403, 335 (2000).

[3] T. S. Gardner, C. R. Cantor, and J. J. Collins, Construction of a Genetic Toggle Switch in Escherichia coli, Nature (London) 403, 339 (2000).

[4] A. E. Friedland, T. K. Lu, X. Wang, D. Shi, G. Church, and J. J. Collins, Synthetic Gene Networks That Count, Science 324, 1199 (2009).

[5] J. S. Markson and M. B. Elowitz, Synthetic Biology of Multicellular Systems: New Platforms and Applications for Animal Cells and Organisms, ACS Synth. Biol. 3, 875 (2014).

[6] A. Adamatzky, Computing in Nonlinear Media and Automata Collectives (CRC Press, Boca Raton, FL, 2001).

[7] M. Cross and H. Greenside, Pattern Formation and Dynamics in Nonequilibrium Systems (Cambridge University Press, Cambridge, England, 2009).

[8] L. Kuhnert, A New Optical Photochemical Memory Device in a Light-Sensitive Chemical Active Medium, Nature (London) 319, 393 (1986).

[9] B. de Lacy Costello, R. Toth, C. Stone, A. Adamatzky, and L. Bull, Implementation of Glider Guns in the LightSensitive Belousov-Zhabotinsky Medium, Phys. Rev. E 79, 026114 (2009).

[10] L. Glass, M. R. Guevara, A. Shrier, and R. Perez, Bifurcation and Chaos in a Periodically Stimulated Cardiac Oscillator, Physica (Amsterdam) 7D, 89 (1983).

[11] M. Courtemanche, L. Glass, and J. P. Keener, Instabilities of a Propagating Pulse in a Ring of Excitable Media, Phys. Rev. Lett. 70, 2182 (1993).

[12] A. Karma, Electrical Alternans and Spiral Wave Breakup in Cardiac Tissue, Chaos 4, 461 (1994).

[13] A. Karma, Spiral Breakup in Model Equations of Action Potential Propagation in Cardiac Tissue, Phys. Rev. Lett. 71, 1103 (1993).

[14] J. N. Weiss et al., From Pulsus to Pulseless: The Saga of Cardiac Alternans, Circ. Res. 98, 1244 (2006).

[15] H. Hsu, E. Huang, X. C. Yang, A. Karschin, C. Labarca, A. Figl, B. Ho, N. Davidson, and H. A. Lester, Slow and Incomplete Inactivations of Voltage-Gated Channels Dominate Encoding in Synthetic Neurons, Biophys. J. 65, 1196 (1993).

[16] R. D. Kirkton and N. Bursac, Engineering Biosynthetic Excitable Tissues from Unexcitable Cells for Electrophysiological and Cell Therapy Studies, Nat. Commun. 2, 300 (2011).

[17] J. Park, C. A. Werley, V. Venkatachalam, J. M. Kralj, S. D. Dib-Hajj, S. G. Waxman, A. E. Cohen, and M. Rota, Screening Fluorescent Voltage Indicators with Spontaneously Spiking HEK Cells, PLoS One 8, e85221 (2013).

[18] R. A. B. Burton, A. Klimas, C. M. Ambrosi, J. Tomek, A. Corbett, E. Entcheva, and G. Bub, Optical Control of Excitation Waves in Cardiac Tissue, Nat. Photonics 9, 813 (2015).

[19] V. Emiliani, A. E. Cohen, K. Deisseroth, and M. Hausser, All-Optical Interrogation of Neural Circuits, J. Neurosci. 35, 13917 (2015).

[20] Q.-T. Nguyen, L. F. Schroeder, M. Mank, A. Muller, P. Taylor, O. Griesbeck, and D. Kleinfeld, An In Vivo Biosensor for Neurotransmitter Release and In Situ Receptor Activity, Nat. Neurosci. 13, 127 (2010).

[21] L. Ye, W. H. Zimmermann, D. J. Garry, and J. Zhang, Patching the Heart: Cardiac Repair from Within and Outside, Circ. Res. 113, 922 (2013).

[22] W. E. Brownell, F. Qian, and B. Anvari, Cell Membrane Tethers Generate Mechanical Force in Response to Electrical Stimulation, Biophys. J. 99, 845 (2010).

[23] See Supplemental Material at http://link.aps.org/ supplemental/10.1103/PhysRevX.6.031001 for Supplementary Figures 1-4 and Supplementary Movies 1-9. 
[24] D. R. Hochbaum et al., All-Optical Electrophysiology in Mammalian Neurons Using Engineered Microbial Rhodopsins, Nat. Methods 11, 825 (2014).

[25] A. Butterweck, U. Gergs, C. Elfgang, K. Willecke, and O. Traub, Immunochemical Characterization of the Gap Junction Protein Connexin45 in Mouse Kidney and Transfected Human HeLa Cells, J. Membr. Biol. 141, 247 (1994).

[26] D. Qin, Y. Xia, and G. M. Whitesides, Soft Lithography for Micro- and Nanoscale Patterning, Nat. Protoc. 5, 491 (2010).

[27] M. Guevara, G. Ward, A. Shrier, and L. Glass, Electrical Alternans and Period Doubling Bifurcations, IEEE Comput. Cardiol. 562, 167 (1984).

[28] I. Banville and R. A. Gray, Effect of Action Potential Duration and Conduction Velocity Restitution and Their Spatial Dispersion on Alternans and the Stability of Arrhythmias, J. Cardiovasc. Electrophysiol. 13, 1141 (2002).

[29] C. Cabo, A. M. Pertsov, W. T. Baxter, J. M. Davidenko, R. A. Gray, and J. Jalife, Wave-Front Curvature as a Cause of Slow Conduction and Block in Isolated Cardiac Muscle, Circ. Res. 75, 1014 (1994).

[30] M. H. Wussling, K. Scheufler, S. Schmerling, and V. Drygalla, Velocity-Curvature Relationship of Colliding Spherical Calcium Waves in Rat Cardiac Myocytes, Biophys. J. 73, 1232 (1997).

[31] V. G. Fast and A. G. Kléber, Role of Wavefront Curvature in Propagation of Cardiac Impulse, Cardiovasc. Res. 33, 258 (1997).

[32] R. R. Aliev and A. V. Panfilov, A Simple Two-Variable Model of Cardiac Excitation, Chaos Solitons Fractals 7, 293 (1996).

[33] F. W. Lindemans and J. J. Denier Van der Gon, Current Thresholds and Liminal Size in Excitation of Heart Muscle, Cardiovasc. Res. 12, 477 (1978).

[34] T. Zaglia et al., Optogenetic Determination of the Myocardial Requirements for Extrasystoles by Cell TypeSpecific Targeting of ChannelRhodopsin-2, Proc. Natl. Acad. Sci. U.S.A. 112, E4495 (2015).

[35] Z. Zhang, Z. Zhao, Y. Liu, W. Wang, Y. Wu, J. Ding, and Z. Zhang, Kinetic Model of Nav1.5 Channel Provides a Subtle Insight into Slow Inactivation Associated Excitability in Cardiac Cells, PLoS One 8, e64286 (2013).

[36] M. E. Gellens, A. L. George, L. Q. Chen, M. Chahine, R. Horn, R. L. Barchi, and R. G. Kallen, Primary Structure and Functional Expression of the Human Cardiac TetrodotoxinInsensitive Voltage-Dependent Sodium Channel, Proc. Natl. Acad. Sci. U.S.A. 89, 554 (1992).

[37] J. P. Keener, A Geometrical Theory for Spiral Waves in Excitable Media, SIAM J. Appl. Math. 46, 1039 (1986).

[38] G. R. Mines, On Circulating Excitations in Heart Muscles and Their Possible Relation to Tachycardia and Fibrillation, Trans. R. Soc. Canada 4, 43 (1914).

[39] L. H. Frame and M. B. Simson, Oscillations of Conduction, Action Potential Duration, and Refractoriness. A Mechanism for Spontaneous Termination of Reentrant Tachycardias, Circulation 78, 1277 (1988).
[40] L. Glass, Y. Nagai, K. Hall, M. Talajic, and S. Nattel, Predicting the Entrainment of Reentrant Cardiac Waves Using Phase Resetting Curves, Phys. Rev. E 65, 021908 (2002).

[41] Y. Nagai, H. Gonzlez, A. Shrier, and L. Glass, Paroxysmal Starting and Stopping of Circulating Waves in Excitable Media, Phys. Rev. Lett. 84, 4248 (2000).

[42] Z. Noszticzius, W. Horsthemke, W. D. McCormick, H. L. Swinney, and W. Y. Tam, Sustained Chemical Waves in an Annular Gel Reactor: A Chemical Pinwheel, Nature (London) 329, 619 (1987).

[43] T. Sakurai, E. Mihaliuk, F. Chirila, and K. Showalter, Design and Control of Wave Propagation Patterns in Excitable Media, Science 296, 2009 (2002).

[44] Z. Qu, F. Xie, A. Garfinkel, and J. N. Weiss, Origins of Spiral Wave Meander and Breakup in a Two-Dimensional Cardiac Tissue Model, Ann. Biomed. Eng. 28, 755 (2000).

[45] M. L. Koller, M. L. Riccio, and R. F. Gilmour, Jr., Dynamic Restitution of Action Potential Duration during Electrical Alternans and Ventricular Fibrillation, Am. J. Physiol.: Heart Circ. Physiol. 275, H1635 (1998).

[46] J. Nolasco and R. W. Dahlen, A Graphic Method for the Study of Alternation in Cardiac Action Potentials, J. Appl. Physiol. 25, 191 (1968).

[47] G. R. Mines, On Dynamic Equilibrium in the Heart, J. Physiol. 46, 349 (1913).

[48] J. N. Weiss, A. Garfinkel, H. S. Karagueuzian, Z. Qu, and P.S. Chen, Chaos and the Transition to Ventricular Fibrillation: A New Approach to Antiarrhythmic Drug Evaluation, Circulation 99, 2819 (1999).

[49] D. Noble, A Modification of the Hodgkin-Huxley Equations Applicable to Purkinje Fibre Action and Pace-Maker Potentials, J. Physiol. 160, 317 (1962).

[50] Y. Wang, J. Mi, K. Lu, Y. Lu, and K. Wang, Comparison of Gating Properties and Use-Dependent Block of Na $v 1.5$ and Na v 1.7 Channels by Anti-Arrhythmics Mexiletine and Lidocaine, PLoS One 10, e0128653 (2015).

[51] H. C. Cho and E. Marban, Biological Therapies for Cardiac Arrhythmias: Can Genes and Cells Replace Drugs and Devices?, Circ. Res. 106, 674 (2010).

[52] G. K. Wang, C. Russell, and S. Y. Wang, State-Dependent Block of Voltage-Gated $\mathrm{Na}+$ Channels by Amitriptyline via the Local Anesthetic Receptor and Its Implication for Neuropathic Pain, Pain 110, 166 (2004).

[53] N. Dascal, Ion-Channel Regulation by G Proteins, Trends Endocrinol. Metab. 12, 391 (2001).

[54] M. E. Todhunter, N. Y. Jee, A. J. Hughes, M. C. Coyle, A. Cerchiari, J. Farlow, J. C. Garbe, M. A. LaBarge, T. A. Desai, and Z. J. Gartner, Programmed Synthesis of Three-Dimensional Tissues, Nat. Methods 12, 975 (2015).

[55] J. M. Kralj, A. D. Douglass, D. R. Hochbaum, D. Maclaurin, and A.E. Cohen, Optical Recording of Action Potentials in Mammalian Neurons Using a Microbial Rhodopsin, Nat. Methods 9, 90 (2012). 